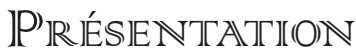

\section{LANGUES EN DANGER ET THÉORIES LINGUISTIQUES : PERSPECTIVES CROISÉES}

\author{
Médéric Gasquet-Cyrus*, Alice Vittrant** et Sylvie Voisin** \\ Aix-Marseille Université, *Laboratoire Parole et Langage, **Dynamique Du Langage
}

La question des « langues en danger » (LED) connaît un essor considérable depuis les années 1990 (voir Gasquet-Cyrus 2010 pour un historique). Prise en charge par des institutions comme l'UNESCO ${ }^{1}$, des fondations internationales ou plus récemment par un projet lancé par Google ${ }^{2}$, elle fait aussi l'objet de nombreuses publications dans un domaine désormais bien établi, où se croisent, au carrefour de différentes disciplines, des questions de vitalité, de droits, de documentation et de revitalisation ${ }^{3}$. Ce champ jouit également d'une couverture médiatique rare en linguistique, avec des publications largement diffusées auprès du grand public, comme Crystal (2000), Hagège (2000), Nettle et Romaine (2000), et plus récemment Evans (2011).

Cette question est souvent posée sur le plan de l'émotion (Harrison 2010) face à la disparition programmée d'une grande partie du patrimoine immatériel de l'humanité, avec un ton dramatique et un appel aux bons sentiments largement

1 Voir notamment l'Atlas des langues en danger; http://www.unesco.org/languages-atlas/

2 www.endangeredlanguages.com

3 Voir aussi entre autres Austin et Sallabank (2011), la 'Cambridge Conference on Endangered Languages' annuelle, les revues Language Documentation and Description et Language Documentation and Conservation. 
critiqués (Calvet 2002, De Swaan 2007, Duchêne \& Heller 2007, Mufwene 2005, Perley 2012). Sommé de se positionner dans ce qui est au moins un débat passionnel (sinon théorique), le linguiste peut aussi et surtout tenter d'apporter une contribution pertinente à partir de recherches concrètes, de travaux empiriques et d'un positionnement (théorique, éthique) réfléchi et assumé

Dans cette optique, ce volume propose de recentrer le débat sur les enjeux théoriques de l'étude des LED, sous l'angle de l'empirisme, au sens que lui donne Auroux (1998). Ce dernier défend l'idée que la linguistique est une science avec observatoire, et non purement spéculative ou expérimentale, l'observatoire étant défini comme « tout instrument qui permet d'observer un phénomène qui ne saurait l'être sans lui » (ibid., p. 166). L'élargissement de l'empirisme à l'observation directe des phénomènes langagiers permet d'intégrer la linguistique de terrain et l'observatoire particulier qu'elle représente. La question qui peut donc être posée, à travers des analyses et des enquêtes de terrain sur les LED, est la suivante : permettent-elles d'observer des phénomènes qui ne sauraient l'être sans elles ? Par ailleurs, si (toujours selon Auroux) les observatoires sont des « objets stables » obtenus par la médiation d'une « instrumentalisation », les LED constituent-elles des « objets stables », ou bien la catégorie même est-elle à interroger, jusqu'à en réfuter la pertinence?

Dans le cadre de ce numéro ${ }^{4}$, ces questions ont été posées par des linguistes qui y ont répondu à partir de leurs disciplines respectives, aux méthodes et aux objectifs différents ${ }^{5}$, avec comme dénominateur commun des enquêtes de terrain. Linguistes descriptivistes, typologues et sociolinguistes, les contributeurs de ce volume interrogent d'un point de vue épistémologique la pertinence de considérer les LED comme un observatoire en soi, et plus largement la contribution des « données » propres aux LED à la construction des théories linguistiques.

Dans cette introduction, nous reviendrons sur l'appellation même de LED. Au vu des différentes contributions, il semble que cette étiquette renvoie à une catégorie construite en discours à différents niveaux, et non à un type de langues. Les LED sont-elles pour autant un « objet stable »? Nous passerons ensuite rapidement sur l'apport des langues nouvellement décrites, LED comprises, qui amène à une meilleure connaissance du langage dans sa globalité et dans ses particularités, avant de nous attarder sur l'impact des LED dans les études typologiques, en particulier dans le domaine de l'échantillonnage et des aires linguistiques. Puis

4 L'idée a germé suite à un atelier présenté lors d'une école thématique du CNRS («Expérience, empiricité, expérimentation en linguistique : histoire et épistémologie »), dans le cadre de la Biennale d'histoire des théories linguistiques ; Agay, 3-7 septembre 2012.

5 Ces croisements sont à l'œuvre dans le cadre du parcours « Langues en Contact et Terrains » du Master en Sciences du Langage de l'Université d'Aix-Marseille, dans lequel interviennent les trois co-éditeurs/-trices de ce volume; https://thelitex.hypotheses.org/lct 
nous explorerons les relations entre LED et diversité linguistique, avant d'en venir aux différents types de locuteurs de ces langues. Enfin, le croisement des regards et de ces champs d'analyse met aussi en exergue la complexité de la notion de langue : nous reviendrons alors, dans une dernière partie, sur les différentes fonctions mises en avant dans les différents articles pour repenser la notion de langue.

\section{LE CROISEMENT DES APPROCHES : DE NOUVELLES PERSPECTIVES?}

La recherche sur les langues en danger s'est principalement développée dans les années 1990 dans la littérature anglophone (Hale \& al 1992, Grenoble \& Whaley 1998), avec au départ une prise de conscience de la « disparition » de nombreuses langues dans le monde perçue comme inquiétante, puis des réflexions sur les causes de ces disparitions et des questionnements sur les communautés de locuteurs, leur construction identitaire et leur pratiques langagières.

La catégorie LED peut s'appréhender à différents niveaux, selon que l'on s'intéresse aux facteurs linguistiques, extralinguistiques et sociaux de l'évolution du paysage linguistique mondial. Mais l'identification des LED comme une catégorie particulière de langue n'est jamais remise en cause, elle semble être considérée par tous comme un " objet stable », évaluable par des critères pourtant changeants (cf. GIDS $^{6}$, Unesco $^{7}$, EGIDS $^{8}$ ). La question posée est ici est savoir si les LED renvoient à une catégorie différente de ce que l'on nomme par ailleurs, sans spécification, « langue ». Autrement dit, est-ce que les LED constituent un type particulier de langues qui offre un champ d'études différent et donc pertinent pour une meilleure compréhension du langage et des langues ? La définition de "langues en danger» repose sur le degré de vitalité qui, nous le verrons dans la section $\S 2.2$, ne recoupe pas nécessairement toujours les mêmes critères. Pour autant, cette variation dans le degré de vitalité fait-elle des LED des langues différentes des autres?

Les contributions de ce volume montrent que la situation des LED conduit à adopter des méthodes d'enquêtes spécifiques, impose des contraintes et crée des obstacles que l'on ne rencontre pas nécessairement avec les autres langues. Mais elles permettent aussi d'observer les phénomènes à travers une autre focale, et de considérer différemment certains aspects linguistiques et sociolinguistiques.

6 Le GIDS (Graded Intergenerational Disruption Scale) a été développé par Fishman (1991).

7 Le questionnaire d'évaluation sur la vitalité des langues en danger de l'UNESCO est issu d'un programme d'aide à la promotion des langues comme instruments d'éducation et de culture. C'est le résultat d'un travail collaboratif qui a réuni des linguistes, spécialistes de langues en danger, plusieurs fois entre 2002-2003. Ce questionnaire est disponible sur le site : http://www.unesco.org/culture/ich/doc/src/00120-EN.pdf

8 Version complétée du modèle de Fishman, l'EGIDS (Extended Graded Intergenerational Disruption Scale) a été développé par Lewis \& Simons (2009). 
Nous revenons donc dans cette section sur l'apport que le croisement des approches descriptives, sociolinguistiques et typologiques apporte à la connaissance des LED et à une meilleure compréhension de cette problématique.

\subsection{LED et typologie}

La description des LED, comme toute nouvelle description de langue, quelle qu'elle soit, contribue à une meilleure connaissance du langage dans sa globalité et dans ses particularités. D'une façon générale, la confrontation d'hypothèses théoriques sur les invariants langagiers à de nouvelles données linguistiques, comme celles que peuvent fournir (aussi) les LED, va permettre la validation de généralisations sur le langage. La prise en compte de ces langues souvent orales et sans tradition grammaticale établie, au statut social rarement prestigieux, contribuera aussi à une connaissance plus juste de la diversité des structures linguistiques. En effet, « il suffit de parcourir n'importe quel ouvrage de typologie pour constater que parmi les phénomènes mentionnés, une bonne proportion est attestée principalement ou même exclusivement dans des langues limitées à des communautés numériquement peu importantes et de ce fait, menacées dans leur existence à plus ou moins brève échéance » (Creissels, ce volume).

Un autre domaine où la prise en compte des LED peut faire progresser la typologie est la question du choix des langues à comparer dans les travaux typologiques. Les choix à opérer dans ce domaine peuvent facilement biaiser les interprétations par le poids donné aux langues parlées par des communautés relativement importantes, ne reflétant pas nécessairement la diversité linguistique. L'ajout de nouvelles descriptions et la connaissance qu'apportent ces descriptions de langues moins standardisées invitent à repenser les comparaisons de langues dans le cadre de travaux typologiques. En particulier, ces ajouts incitent à prendre en compte, dans la constitution des échantillons de langues, les liens génétiques entre ces langues autant que les zones géographiques dans lesquelles elles sont parlées.

Cette question de la construction d'un échantillon de langues représentatif de la diversité langagière reste aujourd'hui centrale en typologie. Les méthodes pour constituer l'échantillon sur lequel le typologue va baser ses hypothèses sont diverses, et dépendent en grande partie du type d'étude menée. Rijkhoff \& Bakker (1998, p. 264-5) proposent de caractériser les échantillons en deux types majeurs, selon que l'on souhaite (1) découvrir la gamme des variations la plus complète pour exprimer un concept dans les langues du monde ('variety samples'), (2) étudier la probabilité d'occurrences d'un phénomène linguistique ('probability samples'). On peut y ajouter une troisième possibilité qui est d'utiliser un échantillon de langues choisies de façon aléatoire et sans critères de tri (génétique, géographique, 
etc.) ('random sample'), avec la nécessité d'un nombre important de langues pour pouvoir produire des résultats et des statistiques fiables (cf. WALS).

Le choix des langues à tester dans le cadre d'une étude typologique est de plus en plus travaillé, afin d'éviter des biais tels que la surreprésentation d'une structure particulière ou d'une famille de langues ${ }^{9}$. Les typologues ont pris conscience de la nécessité de réfléchir à la construction des échantillons, à la taille à leur attribuer (Song 2001, p. 17-38) et à cibler les langues sur lesquelles vont reposer les analyses. Ainsi, ils tiennent compte (1) de la proximité géographique des langues - le contact des langues pouvant induire des convergences, (2) du partage de traits culturels - lequel peut être reflété dans la structure des langues, et bien sûr (3) des relations génétiques ${ }^{10}$. L'accès aux sources peut encore, de façon très pragmatique, conditionner le choix des langues à inclure dans un échantillon, voir par exemple Stolz (2007) sur le problème des corpus parallèles et des traductions accessibles. Nous retiendrons ici que les relations génétiques jouent un rôle important, parfois prioritaire (Rijkhoff \& Bakker 1998) ${ }^{11}$ dans toutes les méthodes d'échantillonnage.

Ces méthodes restent cependant problématiques, elles postulent l'existence de relations génétiques comme allant de soi, comme des données non discutables. On pourrait mettre en regard la classification des langues d'Amérique de Greenberg (1987) et celle de la SIL (Lewis 2009). Voisin (ce volume) fait un tel constat pour l'Afrique, un continent qui atteste le plus de langues au monde, avec un tiers des langues parlées actuellement. Après avoir dressé le bilan des langues menacées et ce malgré un manque flagrant de données pour un grand nombre d'entre elles, elle montre que l'avancée des connaissances sur des zones géographiques particulières ou des familles de langues jusqu'alors peu décrites conduit à des réorganisations importantes des premières classifications. Des enquêtes récentes sur des langues peu décrites ou des LED ont ainsi mis en évidence des apparentements plus distants, l'existence d'un plus grand nombre d'isolats... En d'autres termes, elles redessinent la carte des affiliations. Et Voisin d'ajouter : « [o]n peut donc raisonnablement supposer que l'éclatement en cours dans les affiliations des langues africaines ne pourra qu'augmenter avec l'avancée des travaux de descriptions. »

9 On peut se reporter à Bakker (2011) pour une présentation critique des différentes techniques d'échantillonnage présentées depuis Bell (1978).

10 Rijkhof \& al (1993, p. 172) liste la similarité typologique entre les langues comme un quatrième biais possible, qui peut découler du précédent : des langues génétiquement proches auront tendance à être du même type syntaxique, avec un ordre des mots hérité de la protolangue.

11 «Since languages are assigned to different genetic groups precisely because they differ on a number of linguistic parameters, we have developed a method in which genetic diversity is given precedence over any of the other parameters along which languages can be stratified on which can be a source of bias (such as geographic location). The method provides for maximal genetic distance between languages, [...] First we take into account linguistic variation ACROSS independent language families; then we consider variation WITHIN each individual family ». (Rijkhoff \& Bakker 1998, p. 267-9) 
La description de LED peut donc remettre en cause de façon sérieuse le classement génétique des langues d'une région et amener à une révision des classifications. Or, étant donné l'importance des relations génétiques dans le choix des échantillons de langues en typologie, cette restructuration des familles de langues a un impact non négligeable sur les études typologiques. On retiendra ici l'idée développée par Dahl (2008) d'une méthode d'échantillonnage des langues a posteriori (plutôt qu' a priori) qui aurait l'avantage de tenir compte de ces évolutions pour le calcul de la diversité linguistique et pour une meilleure représentativité des langues. Il suggère de prendre en compte d'autres facteurs pour mesurer la diversité linguistique ${ }^{12}$ et structurelle entre les langues et construire un échantillon plus représentatif de cette diversité. Il mentionne la taille de la communauté, le degré d'alphabétisation ('literacy') et la pression de la géographie sur la densité de population.

Ce dernier critère de la pression aréale semble particulièrement pertinent. Il prend en compte le fait que des langues non apparentées peuvent attester une diversité faible, alors que des langues d'une même famille peuvent montrer une distance typologique supérieure à la moyenne, comme c'est le cas pour le zoulou et le yoruba, deux langues Niger-Congo ou entre le khmer et le mundari, deux langues austro-asiatiques (Dalh 2008, p. 214, 220). En conséquence, relever du même phylum ne garantit pas une similarité typologique. Et si l'on souhaite construire un échantillon de langues le plus diversifié possible, le plus représentatif de la diversité de langues, il est important (1) de tenir compte de la distance typologique entre les langues, quelle que soit la méthode utilisée pour calculer cette distance, mais aussi (2) d'intégrer le facteur de pression aréale, car la diversité structurelle peut se révéler moindre entre des langues non-affiliées du fait de leur appartenance à une aire linguistique.

Relation génétique et diversité linguistique jouent donc un rôle important dans la construction des échantillons de langues utilisés dans les études typologiques. Les LED, dans ces travaux typologiques, méritent alors une attention particulière, en raison d'une part de la prise en compte nécessaire des remises en cause de certaines parentés de langues ou de restructuration interne de certains phylums ; d'autre part, les LED amènent à changer notre regard sur la diversité linguistique réelle entre les langues apparentées ou non-apparentées. Un échantillonnage a posteriori comme proposé par Dahl (2008) semble intéressant dans le cas de l'insertion des LED dans les travaux typologiques, puisque la domination sociale dans laquelle se trouvent les locuteurs des LED constitue généralement un terrain propice aux interférences linguistiques.

12 Sur différentes approches du calcul de la diversité linguistique ou des différences entre systèmes langagiers, voir aussi Borin (2013). 


\subsection{LED et diversité}

La « diversité » est une caractéristique mise en avant dans la problématique liée aux LED.

As the drama is repeated over and over the around the world, it is easy to forget a crucial feature of the phenomenon: the very diversity that makes the loss irreversible. (Mithum 1998, p. 163)

D'autres motifs de préoccupation sont également apparus au cours des années quatre-vingt-dix, concernant les préjudices que peuvent subir les personnes qui parlent des langues menacées, ainsi que l'humanité tout entière, sous l'effet de l'érosion de la diversité linguistique dans le monde. (Maffi 2002, p. 426)

La disparition des langues est souvent associée à la perte d'autres éléments et corrélée à une perte plus large de la diversité. La perte de plusieurs types de diversité associée à la disparition des langues est ainsi évoquée, voire invoquée pour préserver les LED. L'inventaire des diversités citées permet de voir que la notion de diversité doit être envisagée, dans la problématique des LED, sous différents angles.

Un des apports de ce volume, sans pour autant juger de la pertinence de ces corrélations, est de mettre une certaine distance entre d'une part, l'urgence pour les linguistes de décrire et documenter et d'autre part, les situations diverses que vivent les locuteurs. Tout d'abord, les communautés n'ont pas nécessairement les mêmes attentes malgré la « menace » qui pèse sur leur langue. Toutes les contributions de ce volume montrent qu'il y a une différence nette entre description, documentation et revitalisation. Ces distinctions sont parfois masquées par le terme générique de sauvegarde. La question de ce qui est sauvegardé est notamment posée dans ce volume par Pivot (cf. en particulier la problématique liée aux néolocuteurs, ce point particulier des compétences et des locuteurs sera développé plus loin).

La diversité peut également être évoquée comme critère pour évaluer le degré de vitalité des langues, parfois liée explicitement ou implicitement avec l'urgence de documentation. On ne compte plus désormais les listes et les cartes qui répertorient les LED avec des codes différents pour identifier le degré de menace ( « en danger », « moribonde », « vulnérable », « threatened », " severely endangered $\gg . .$.$) . Sous chacune de ces codifications, des critères d'évaluation du$ degré de vitalité des langues sont posés. Ainsi, en prenant l'exemple de Livingtongues $(2007)^{13}$, les language hotspots ne sont pas seulement définis à l'aide de critères qui permettent de connaître le degré de vitalité pour les langues de la zone, ils incluent dans leur calcul d'urgence pour la documentation, le degré de 
diversité génétique, i.e. le nombre de familles linguistiques différentes dans la zone. Pour autant, prendre en compte la diversité génétique dans des aires géographiques particulières, comme le fait Livingtongues, ne permet pas de décrire des langues qui seront nécessairement éloignées typologiquement. Comme cela a été indiqué dans la section précédente, les langues d'une même famille peuvent avoir des caractéristiques différentes en raison d'une influence aréale. Les structures " exotiques » sur le plan typologique peuvent également être l'innovation d'une seule langue à l'intérieur d'une famille. On pourrait mentionner l'exemple de l'anglais, qui avec les langues papoues constitue l'une des rares langues connues pour utiliser le verbe faire (do) pour former des propositions interrogatives et négatives (cf. Voisin, ce volume).

Un autre écueil possible, lorsque l'on focalise l'attention sur de telles zones pour mieux appréhender la diversité structurelle, est la proximité géographique. Cette dernière aboutit souvent à des phénomènes d'interférence dus au contact, notamment dans les situations de bilinguisme et de domination dans lesquelles se trouvent les LED. Ces situations réduisent les chances de découvrir de nouvelles structures par le biais de ces interférences. Comme le note Evans (2011), la linguistique n'a pas de pouvoir de déduction. La nature des traits typologiques découverts par la description des langues ne peut être anticipée, pas plus que les langues dans lesquelles ces traits peuvent être trouvés.

Pour autant, cette corrélation entre LED et diversité linguistique ne doit pas être rejetée en bloc. Les LED sont reconnues comme un observatoire potentiel pour la typologie (cf. Creissels, ce volume), mais au même titre que les autres langues.

Les particularités de ce volume font également émerger un autre type de diversité interne à la problématique des LED, à savoir la diversité des méthodes et des approches appliquées par les linguistes aujourd'hui sur ces langues. En effet, les analyses et les terrains peuvent avoir des finalités assez différentes. L'objectif et la méthode peuvent être purement descriptifs, liés uniquement à la documentation ou à la revitalisation de la langue, ou bien les trois à la fois. Dans le cadre d'une approche descriptiviste, les LED interrogent les données ainsi recueillies et leur place dans une typologie des langues (cf. Creissels, Voisin, ce volume). Certaines précautions doivent être prises pour identifier au mieux la langue sur laquelle le travail est en train d'être réalisé. Creissels met également en garde sur les pans de la grammaire que le linguiste ne pourra décrire dans le cas des langues moribondes. Les mêmes restrictions valent pour la documentation, lorsque certains savoir-faire ont d'ores et déjà disparu.

La diversité des communautés, des situations politiques et sociales dans lesquelles les LED évoluent et la diversité des profils de locuteurs sont abordées dans ces 
différentes approches et par conséquent dans les différents articles de ce volume. Elles sont bien évidemment plus prégnantes dans les approches sociolinguistiques, mais si elles ne sont pas l'objet de la recherche dans les tâches de description, elles ne sont pas pour autant ignorées des descripteurs. Il suffit de reprendre les différents ouvrages de méthodes pour le travail de description sur le terrain pour voir l'importance qu'attachent les descripteurs à la recherche des locuteurs. On peut renvoyer sur ce point à Mithun 2001, p. 48 sq. - « Who shapes the record : speaker and linguist », qui consacre une section aux locuteurs. Là aussi, comme le met en avant Rangel (ce volume), certains informateurs seront privilégiés pour des types de tâches particuliers, comme le notent aussi Newman \& Ratliff (2001).

As a number of the authors have noted [...], some speakers have special talents which the alert fieldworker will recognize and utilize. [...] I [Ratliff] worked initially with three speakers of Hmong in my fieldwork. One had perfectly clear articulation, and was an ideal model for the impressively complex sound system of the language, although he appeared to have no interest in his own language or in the research itself. Another was no more a native linguist than the first (and he had a lisp), but was a fine native anthropologist, and could hold forth at length about cultural components of the language. The third was a natural linguist: he could understand the purpose of the research and produce exactly what I requested and, moreover, he could find patterns in the data independently. (Newman \& Ratliff 2001, p. 4)

Pour les champs plus spécifiques à la sociolinguistique, la diversité des regards portés sur les LED par les locuteurs et les non-locuteurs conduit à introduire de la diversité sous l'appellation même de LED. Cette prise en compte de la diversité des LED elles-mêmes et de leurs locuteurs est une question centrale dans toutes ces approches et méthodes, même si elles ne sont pas nécessairement envisagées sous le même angle.

\subsection{LED et locuteurs}

Les recherches sur les LED ont eu le mérite de mettre l'accent sur la problématique des locuteurs. En effet, au $\mathrm{Xx}^{\mathrm{e}}$ siècle, la linguistique s'est développée autour de la construction d'un objet langue, un artefact, une « fiction utile » comme le disait Einar Haugen : « The concept of language as a rigid, monolithic structure is false, even if it has proved to be a useful fiction in the development of linguistics » (1971, p. 24). Ce qui justifie qu'un sociolinguiste comme Calvet demande : La langue est-elle une invention des linguistes $?^{14}$ Certes, les années 1960 avaient déjà vu l'essor de domaines moins axés sur la langue en elle-même et pour elle-même que sur les sujets parlants : linguistique de l'énonciation, pragmatique, analyse

14 Cette question est le sous-titre proposé par Calvet pour son livre Essais de linguistique (2004). 
conversationnelle, analyse interactionnelle, analyse de discours, et bien sûr sociolinguistique. Mais ces disciplines ne travaillent finalement que sur des parties, des niveaux de "la langue » : énoncés, interactions, textes, discours, variétés...

Or, la question des LED a été posée en prenant en compte les langues dans leur globalité, à la fois en tant que (i) systèmes de communication dotés d'une grammaire et en tant qu'(ii) institutions sociopolitiques. Dire que la langue $x$ est en danger revient à dire que (i) le système est susceptible de ne plus être utilisé, ou d'être modifié, changé, altéré par des pratiques en recul, des phénomènes d'attrition, etc. et/ou que (ii) cette langue est minorisée, voit son statut et son éventuel prestige décroître, au point d'être délaissée par les locuteurs et de moins en moins transmise. Tout ceci rend la question du locuteur et du « bon » locuteur cruciale. Mais cette vision macro, très pratique quand il s'agit de cartographier des langues en danger ou d'alerter le grand public, ne résiste pas à des approches plus micro, plus ethnographiques.

Quand ils vont sur le terrain, au lieu d'envisager de manière globale des locuteurs relativement anonymes des langues $x$ ou $y$, certains linguistes se rendent bien compte que les locuteurs ne possèdent pas les mêmes compétences, qu'il s'agisse de traduire, de prononcer, de répondre à des tâches d'élicitation en produisant des constructions pertinentes ou de fournir des explications métalinguistiques (voir ci-dessus la citation de Newman \& Ratliff 2001). Plus précisément, dans des processus d'attrition ou de perte des fonctions communicatives, les locuteurs des LED ne sont pas au même «niveau » : là où certains sont encore capables de produire des discours complets et des constructions complexes dans la langue, d'autres ne peuvent qu'en prononcer quelques fragments ; certains sont capables de l'utiliser uniquement dans certains contextes ou genres discursifs, alors que d'autres disposent d'une palette stylistique plus large ; certains la comprennent intégralement, alors que d'autres n'en saisissent plus que quelques bribes, ce qu'expliquent bien Bert et Grinevald (2010) :

Dans les communautés linguistiques de langues menacées, la variété des
locuteurs est encore plus importante. Deux spécificités expliquent cette plus
grande diversité. En premier lieu, le nombre de personnes au profil atypique est
plus élevé, car les monolingues dans la langue menacée sont peu nombreux et
la proportion de bilingues, de différents niveaux de compétence, est importante.
De surcroit, les situations de LED sont caractérisées par la présence de locuteurs
aux profils particuliers, présentant souvent des traits socio-psychologiques
spécifiques. (Bert \& Grinevald 2010, p. 117)

Certains linguistes de terrain prennent donc en considération les caractéristiques des personnes qui ne sont justement pas de simples locuteurs qui parleraient tous la langue au même niveau ; ils s'interrogent sur ce que signifie être (ou ne pas/plus être) un « locuteur » d'une langue en danger. On s'intéresse ainsi aux profils des 
locuteurs, en fonction de leur âge, des conditions dans lesquelles ils ont acquis ou appris la langue, de leur statut social, de leur rapport à la langue, de leurs attitudes, et plus largement de leur biographie langagière.

La question de la compétence est donc déplacée : il ne s'agit plus de dire que tel locuteur serait un " meilleur » informateur parce qu'il maitriserait mieux la langue, mais de prendre en compte tous les acteurs rencontrés, quels que soient leurs profils, car chacun d'eux est une manifestation de « la langue ». En effet, lorsqu'on a affaire à une langue qui n'est plus «parlée » que par une poignée de locuteurs, chacun d'entre eux devient soudain beaucoup plus précieux, avec toutes ses caractéristiques, qu'il s'agisse des variations dans les usages ou dans le statut des locuteurs. Bien sûr, tout dépend des objectifs de la recherche, et Creissels a raison de mettre en garde contre des informateurs aux compétences « fragiles » sur lesquels on se baserait, sans recul, pour la description d'une langue. Cependant, plus encore dans une approche sociolinguistique, surtout sur de petits groupes, on ne sélectionne pas les «bons » informateurs, mais on observe l'usage (exclusif, partiel, déclaré) des langues comme des pratiques langagières à part entière. C'est ainsi que dans son travail sur le zoque au Mexique, Rangel peut établir des nuances très fines entre ses trois principaux interlocuteurs. Comme le laisse entendre Pivot, cela remet en question le mythe du locuteur « natif » qu'il faudrait trouver pour avoir accès à « la langue », d'autant que les LED sont parfois apprises et investies de valeurs identitaires par des locuteurs qui ne l'avaient pas en L1. De nombreux travaux portent aujourd'hui sur le statut de ces néo-locuteurs (O'Rourke, Pujolar et Ramallo 2014 ; Costa 2015).

C'est face à ces réalités du terrain que les linguistes en sont venus à proposer, depuis les semi-locuteurs de Dorian (1977), plusieurs typologies des locuteurs, comme Bert et Grinevald (2010) qui présentent quelques « profils typiques des locuteurs de LED»: les « locuteurs traditionnels », les « semi-locuteurs », les « sous-locuteurs », les « anciens locuteurs », les « locuteurs fantômes », les « néo-locuteurs », les « derniers locuteurs » (et les discours « mythiques » qui les accompagnent)... Dans ce volume, Rangel propose de croiser les typologies des linguistes avec les typologies des locuteurs eux-mêmes, ce qui lui permet de mieux décrire ce qui se joue autour de l'apayaneco. Chaque terrain révèle des profils spécifiques, mais de telles typologies permettent de prendre en compte de la diversité des compétences et des usages des langues. En effet, on ne saurait, par exemple, parler de « communauté linguistique » pour telle langue, alors qu'au sein $\mathrm{du}$ groupe se trouvent des sujets qui correspondent à des profils de locuteurs très différents. L'apparente homogénéité des communautés doit laisser place à la prise en compte de l'hétérogénéité des pratiques langagières et des discours épilinguistiques des locuteurs sur les langues (Rangel, ici-même, préfère d'ailleurs parler de 
《 sous-réseaux ») : ce que les linguistes ne devraient pas ignorer de manière générale est indispensable sur le terrain des LED. Loin des modèles macro qui essaient de donner des indices de la vitalité des langues en croisant des données multiples mais souvent trop générales, imprécises, datées, mal évaluées..., les approches de terrain permettent de voir à quel point cette « vitalité » n'est pas réductible à des chiffres ou à des cases. Là où les grands modèles parlent des langues de manière globale, sur le terrain se pose de manière saillante la question « que veut dire parler une langue? ». Cette question va bien au-delà de la simple compétence, et permet d'interroger la notion même de langue.

\section{ET LA LANGUE?}

On l'a dit plus haut, la notion de langue peut renvoyer à deux modalités : la langue en tant que système linguistique dont on décrit les éléments et le fonctionnement (la grammaire) et la langue en tant qu'institution sociopolitique. Dire « le français est une langue à ordre SVO » ne se situe pas sur le même plan que si l'on dit « le français est une langue importante de la francophonie ». Le premier usage fait référence au corpus de la langue, l'autre à son status, pour reprendre la terminologie de Kloss (1969). De quoi parle-t-on quand on parle de « langue » en danger? Du système linguistique, qui subit des changements profonds en raison d'une baisse du taux de pratique par des usagers habituels ? Ou du statut de la langue, qui décline en même temps que le statut du groupe auquel elle est associée ? Des deux souvent, étant donné qu'il y a un lien entre la perte de statut d'une langue, sa minorisation, et la baisse de sa pratique au profit d'une autre langue (language loss, language shift). Mais dans les deux cas, on se focalise sur la langue alors que ce sont les locuteurs et les sociétés qui sont en premier lieu concernés.

En effet, de « quoi » une langue serait-elle en danger ? De ne plus être parlée ? Cela peut résulter du choix d'un groupe, qui, pour différentes raisons, " préfère » utiliser une autre langue, un autre répertoire. Ainsi, certains groupes sont socialement et politiquement minorisés en raison (entre autres) de leur pratique d'une langue mal 'cotée' sur le marché des langues (Calvet 2002). De fait, ils n'ont pas accès à certaines ressources (emploi, diplômes, culture...), donc pas accès au pouvoir. Ils font donc le choix de recourir à une autre langue. Certes, ce choix est contraint par des pressions sociales et politiques, par les relations de pouvoir entre les groupes qui engendrent un « prestige » plus grand de la langue de certains sur la langue des autres ${ }^{15}$. Mais c'est la réalité du fonctionnement des langues à

15 Le choix porte sur la langue à transmettre, des langues à pratiquer, ceci ne signifie pas que les locuteurs soient pleinement conscients du devenir des langues laissées de côté ou ainsi minorées, ce sont là des problématiques qui parfois ne concernent que le linguiste (Bassène 2010). 
l'échelle mondiale : il n'y a que dans les fantasmes d'un monde sans conflits que les langues vivent en harmonie, il n'y a que dans les utopies des moralistes que toutes les langues du monde doivent être conservées. Aucun linguiste, à notre connaissance, ne « souhaite » la disparition des langues. Mais cette attitude amène parfois certains à vouloir préserver coûte que coûte toutes les langues, quitte à les mettre sous « perfusion » ou dans des « réserves linguistiques » (De Swaan 1999). Vouloir sauver les langues à tout prix part assurément d'un bon sentiment, mais cela va à l'encontre des changements linguistiques qui traversent l'histoire des langues du monde, et surtout de la volonté de groupes qui décident, avec plus ou moins de liberté, avec plus ou moins de contraintes, de conserver ou non l'usage de leur langue.

Cette question éthique - faut-il « sauver » la langue ? - se pose à tout linguiste de terrain. Mais comme cela a été précédemment rappelé, les objectifs doivent être clairement posés entre le chercheur et le groupe sur et avec lequel il travaille : est-il là pour décrire la langue, la documenter ou bien pour aider la communauté à la préserver, la développer ? S'il choisit - et c'est son droit - de travailler sur une langue en danger pour des raisons scientifiques uniquement (avec tout l'intérêt développé plus haut), il se doit cependant de prendre en compte le contexte et les locuteurs dans leur hétérogénéité, que ce soit pour en expliquer la structure (les variations, les changements) ou les pratiques langagières hétérogènes. Ce qui, dans une approche sociolinguistique, revient à dire que « la langue » ne devrait pas être prise comme un tout homogène, réduite à une (et une seule) grammaire, mais au contraire présente sous différentes formes, celles que pratiquent les anciens et/ ou les nouveaux locuteurs, et tous ceux qui correspondent aux profils intermédiaires des typologies évoquées plus haut.

L'attention devra aussi être portée sur le fonctionnement de la langue qui, en perdant tout ou partie de ses fonctions de communication, n'en demeure pas moins une « vraie » langue : le travail de Pivot sur l'importance de la fonction sémiotique des LED va dans ce sens. Elle montre que les LED peuvent illustrer de nouvelles modalités d'existence des langues, au-delà des fonctions communicatives.

\section{POUR CONCLURE}

Typologues et sociolinguistes se rejoignent dans ce volume pour interroger leurs descriptions en regard de la problématique des LED. Les auteurs illustrent chacun la pertinence, dans leur domaine respectif, à considérer les LED comme un observatoire en soi, et montrent comment terrain(s) et théorie(s) se rejoignent dans le domaine des LED. Cependant, les spécificités de chacun et plus largement la contribution de leurs « données » conduisent à la description de situations variées. 
Ce volume s'ouvre par des contributions de typologues qui apportent une vision globale de la place des LED dans les travaux portant sur la recherche des universaux. Si l'importance de décrire les langues menacées pour les travaux typologiques est reconnue de tous, Creissels détaille l'intérêt de cette prise en compte dans ces recherches, mais alerte également sur les conséquences qui découleraient de l'insertion de langues moribondes dans ces analyses typologiques. L'article suivant de Voisin focalise sur la place des langues africaines dans la problématique des LED. Jusque-là, ce continent n'était pas dans son ensemble considéré comme prioritaire : des langues de la région sont certes menacées à brève échéance, mais leur (faible) nombre comparativement à d'autres continents atténue l'urgence de sauvegarde, la diversité linguistique étant préservée. Mais Voisin, dans son article, pose un autre regard sur cette situation et montre notamment que (1) la méconnaissance de la situation réelle des langues de ce continent et (2) des changements récents liés notamment à l'urbanisation et à l'émergence d'une classe moyenne obligent à reconsidérer la position de l'Afrique dans la problématique des LED. Toujours en Afrique, mais avec cette fois une orientation plus sociolinguistique, Boutché et Kplogu détaillent la situation de deux langues, le toyúgbe, une variété de l'éwé parlée au Ghana, et la variété véhiculaire de l'adamawa, une variété du peul parlée au Cameroun. Ils observent notamment l'impact que peut avoir la standardisation et la véhicularisation de langues à la fois sur le plan de la diversité linguistique, comme dans les deux articles précédents, mais aussi sur des problématiques d'identité. Puis, c'est au tour de Pivot de mettre en regard la situation des Rama au Nicaragua avec celle des locuteurs du francoprovençal en France. La situation particulière de ces langues implique des analyses qui ne peuvent être conduites que par l'observation des pratiques sociales. La présentation de ces langues très menacées lui permet d'interroger la fonction des langues et plus largement les notions de communauté linguistique et de langue revue, pour cette dernière, à travers sa fonction sémiotique. Enfin, toujours autour des langues moribondes, Rangel revient sur les locuteurs de LED eux-mêmes. L'originalité de son travail est de croiser les typologies des locuteurs proposées par différents linguistes avec les définitions proposés par ses propres informateurs lors de ses différents terrains sur l'ayapaneco au Mexique. Il ressort de cette confrontation une certaine dynamique dans les différents types de locuteurs mis en évidence à travers les deux points de vue.

Au final, ce volume proposera, à travers des approches croisées, de répondre modestement à la question initiale. Si la catégorie « langue en danger » mérite d'être déconstruite et critiquée en regard de la multiplicité des critères qui lui sont appliqués et des finalités des uns et des autres, entre volonté de « sauvegarde » et « curiosité scientifique », il n'en demeure pas moins que ces « objets », de 
par leur fragilité et leurs situations sociopolitiques particulières, permettent de réviser certaines descriptions linguistiques et de poser des questions sociolinguistiques pertinentes. En cela, les LED semblent constituer un observatoire qui offre l'opportunité de pratiquer une linguistique empirique à même de contribuer à un certain renouvellement théorique.

\section{RÉFÉRENCES}

Auroux, Sylvain, 1998. La raison, le langage et les normes, Paris, Presses Universitaires de France.

Austin, Peter K. \& Sallabank, Julia, 2011. The Cambridge Handbook of Endangered Languages, Cambridge, Cambridge University Press.

Bakker, Dik, 2011. « Language Sampling », Jae Jung Song (ed.), Handbook of Linguistic Typology, Oxford, Oxford University Press

Bassene, Alain-Christian, 2010. «Perte de vitalité du jóola banjal (Sénégal) : révélations d'un linguiste locuteur », Faits de langues 35/36, 363-372.

Bell, Alan, 1978. «Language samples », Joseph H. Greenberg et al. (eds.), Universals of Human Languages: method - theory, vol. 1, Stanford, Stanford University Press, 123156.

Bert, Michel \& Grinevald, Colette, 2010. «Proposition de typologie des locuteurs de LED », Faits de langues 35/36, 117-132.

Borin, Lars, 2013. «The why and how of measuring linguistic differences », Lars Borin \& Anju Saxena (eds.), Approaches to measuring linguistic differences, Berlin, Mouton de Gruyter, 3-25.

Burling, Robbins, 1969. « Proto-Karen: A reanalysis », Occasional Papers of the Wolfenden Society on Tibeto-Burman Linguistics 1, 1-116.

Calvet, Louis-Jean, 2002. Le Marché aux langues: les effets linguistiques de la mondialisation, Paris, Plon.

- 2004. Essais de linguistique : la langue est-elle une invention des linguistes ?, Paris, Plon.

Costa, James, 2015. « New Speakers, New Language: On Being a Legitimate Speaker of a Minority Language in Provence », International Journal of the Sociology of Language 231, 127-145.

Crystal, David, 2000. Language death, Cambridge, Cambridge University Press.

Dahl, Östen, 2008. «An exercise in a posteriori language sampling », Sprachtypologie und Universalienforschung 61, 208-220.

De Swaan, Abram, 2007. « Le sentimentalisme des langues. Les langues menacées et la sociolinguistique ", Michael Werner (ed.), Politiques et usages de la langue en Europe, Paris, Éditions de la Maison des sciences de l'homme (Dialogiques), 82-98.

Dorian Nancy C., 1977. «The Problem of Semi-Speaker in Language Death », International Journal of the Sociology of Language 12, 23-32.

Duchêne, Alexandre \& Heller, Monica, 2007. Discourse of Endangerment: Ideology and Interest in the Defense of Languages, Londres, Continuum (Advances in Sociolinguistics).

Evans, Nicholas, 2011. Dying Words: Endangered Languages and What They Have to Tell Us, Oxford, John Wiley \& Sons.

Fishman, Joshua A., 1991. Reversing Language Shift: Theoretical and Empirical Foundations of Assistance to Threatened Languages, Bristol, Multilingual Matters.

Gasquet-Cyrus, Médéric, 2010. "La 'mort' des langues : repères historiques, critiques et réflexions éthiques ", Médéric Gasquet-Cyrus, Alain Giacomi, Yvonne Touchard, Daniel Veronique (éd.), Pour la (socio)linguistique, Paris, L'Harmattan, 285-296. 
Greenberg, Joseph H., 1987. Language in the Americas, Stanford, Stanford University Press.

Grenoble, Lenore A. \& Whaley, Lindsay J., 1998. « Toward a typology of language endangerment », Lenore Grenoble \& Lindsay Whaley (eds.), Endangered Languages: Current Issues and Future Prospects, Cambridge, Cambridge University Press, 22-54. Hagège, Claude, 2000. Halte à la mort des langues, Paris, Odile Jacob.

Hale, Ken, Krauss, Michael, Watahomigie, Lucille J., et al., 1992. «Endangered languages », Language 68(1), 1-42.

Harrison, David K., 2010. « The Last Speakers », National Geographic.

Haudricourt, André-Georges, 1946. "Restitution du Karen commun », Bulletin de la Société de Linguistique de Paris 42(1), 103-111.

Haugen, Einar, 1971. «The ecology of language », The Linguistic Reporter (supplément 25). 19-26.

Kloss, Heinz, 1969. Research possibilities on group bilingualism: a report, Québec, CIRB. Lewis, Paul M., 2009. Ethnologue: languages of the world. [15th edition], Dallas (Texas), SIL International.

Lewis, Paul M. \& Simons, Gary F., 2009. «Assessing Endangerment: Expanding Fishman's GIDS », Revue roumaine de linguistique 2, 103-120

Maffi, Luisa (ed.), 2001. On biocultural diversity: linking language knowledge and the environment, Washington DC, Smithsonian Institution Press.

— 2002. «Langues menacées, savoirs en péril », Revue internationale des sciences sociales 173. $425-433$.

Mithun, Marianne, 1998. "The significance of diversity in language endangerment and preservation ", Grenoble, L.A. \& Whaley, L.J. (eds.), Endangered Languages; Language Loss and Community Response, Cambridge, Cambridge University Press, 163-191.

— 2001. « Who shapes the record: the speaker and the linguist », Paul Newman \& Martha Ratliff (eds.), Linguistic fieldwork, Cambridge, Cambridge University Press, 34-54.

Mufwene, Salikoko S., 2005. Créoles, écologie sociale, évolution linguistique, Paris, L'Harmattan.

Nettle, Daniel \& Romaine, Suzanne, 2000. Vanishing Voices: The Extinction of the World's Languages: The Extinction of the World's Languages, Oxford, Oxford University Press.

Newman, Paul \& Ratliff, Martha, 2001. Linguistic Fieldwork, Cambridge, Cambridge University Press.

O'Rourke, Bernadette, Pujolar, Joan \& Ramallo, Fernando, 2015. « New Speakers of Minority Languages: The Challenging Opportunity », International Journal of the Sociology of Language 231, 1-20.

Perley, Bernard, 2012. "Zombie Linguistics: Experts, Endangered Languages and the Curse of Undead Voices », Anthropological Forum 22(2), 133-149.

Rijkhoff, Jan \& Bakker, Dik, 1998. «Language sampling », Linguistic Typology 2-3, 263314.

Rijkhoff, Jan, Bakker, Dik, Hengeveld, Kees \& Kahrel, Peter, 1993. " A Method of Language Sampling », Studies in Language 17(1), 169-203.

Song, Jea Jung, 2001. Linguistic Typology. Morphology and Syntax, Harlow, Longman.

Stolz, Thomas, 2007. « Harry Potter meets Le petit Prince - On the usefulness of parallel corpora in crosslinguistic investigations », Berlin, STUF 60(2), 100-117. 